\title{
Identification of TYR mutations in patients with oculocutaneous albinism
}

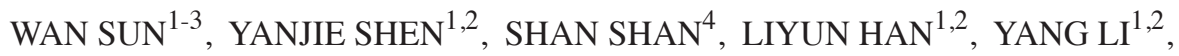 \\ ZHENG ZHOU ${ }^{4}$, ZILIN ZHONG ${ }^{1,2}$ and JIANJUN CHEN ${ }^{1,2}$
}

\author{
${ }^{1}$ Department of Ophthalmology, Shanghai Tenth People's Hospital, Tongji Eye Institute; ${ }^{2}$ Department of Medical Genetics, \\ Tongji University School of Medicine, Shanghai 200092; ${ }^{3}$ Department of Pediatric Ophthalmology, The Second Affiliated Hospital \\ of Nanchang University, Nanchang, Jiangxi 330006; ${ }^{4}$ National Laboratory of Biomacromolecules, \\ Institute of Biophysics, Chinese Academy of Sciences, Beijing 100101, P.R. China
}

Received January 17, 2017; Accepted August 1, 2017

DOI: $10.3892 / \mathrm{mmr} .2018 .8881$

\begin{abstract}
Oculocutaneous albinism (OCA) is a set of autosomal recessive disorders characterized by hypopigmented hair, skin and eyes. Homozygous or compound heterozygous mutations in the tyrosinase (TYR) gene can cause OCA1, which is the most common and severe subtype of albinism. In the present study, 17 patients with non-syndromic OCA were enrolled from eight provinces of China and were non-consanguineous, with the exception of Patient 4000301. Total genomic DNA was isolated from peripheral blood. Screening was performed for the whole exons and their flanking regions of the TYR gene using Sanger sequencing and the pathogenicity of variants was predicted using in silico analysis. In total, 12 TYR mutations were identified in 10 patients, respectively. Of these, two patients carried homozygous mutations and eight patients carried compound heterozygous mutations. Among the 12 TYR mutations, two missense mutations c.1198T $>$ G (p.W400G) and c.819G $>$ T (p.Q273H) were novel. The results of the present study expand the mutation spectrum of the TYR gene, which may further assist in the prenatal examination and genetic diagnosis of OCA.
\end{abstract}

\section{Introduction}

Oculocutaneous albinism (OCA) is a set of autosomal recessive disorders characterized by a reduction or complete absence of melanin in the skin, hair and eyes. The affected individuals present with signs and symptoms including photophobia, nystagmus, poor visual acuity and iris transillumination $(1,2)$.

Correspondence to: Dr Zilin Zhong or Professor Jianjun Chen, Department of Ophthalmology, Shanghai Tenth People's Hospital, Tongji Eye Institute, Tongji University School of Medicine, Medical School Building, 1239 Siping Road, Yangpu, Shanghai 200092, P.R. China

E-mail: zhongzl@tongji.edu.cn

E-mail: chenjianjun@tongji.edu.cn

Key words: autosomal recessive inheritance, oculocutaneous albinism, tyrosinase genes, mutation
No effective therapy for OCA has been found previously. The worldwide prevalence of all known forms of OCA is estimated to be 1:17,000 (3). In the Chinese Han population of Shandong, the prevalence is $\sim 1: 18,000$ and $3.80 \%$ of the population are carriers (4).

To date, mutations in at least 16 genes have been reported to be responsible for OCA (5). The nosology of OCA is based on classification with genetic defects in molecules, comprising 12 syndromic OCA genes and seven non-syndromic OCA genes or loci, including OCA1 (MIM 203100), OCA2 (MIM 203200), OCA3 (MIM 203290), OCA4 (MIM 606574), OCA5 (MIM 615312), OCA6 (MIM 113750) and OCA7 (MIM 615179).

Mutations in both alleles of the tyrosinase (TYR) gene can cause OCA1, which is characterized by a complete or incomplete lack of melanin. OCA1 is the most predominant subtype in the Chinese Han population (5). OCA2, associated with mutations in the OCA2 gene, is the most common type of albinism in the black African population (6). OCA3 is rare in Asiatic populations associated with tyrosinase-related protein 1 (TYRP1). OCA4, OCA6 and OCA7 are caused by mutations in SLC45A2, SLC24A5 and C10 or f11, respectively. OCA5 is a novel genetic cause mapped to chromosome 4q24 (7).

As TYR mutations are responsible for $70.1 \%$ of cases of OCA in the Chinese population (5), the present study performed direct sequencing of TYR in 17 patients with OCA, which revealed 12 mutations in 10 patients, respectively, including two novel mutations c.1198T $>$ G (p.W400G) and c.819G>T (p.Q273H).

\section{Patients and methods}

Patients and clinical data. A total of 17 patients with OCA were enrolled in the present study, including 6 women and 11 men, who were from 8 provinces of China (Beijing, Jiangsu, Hebei, Liaoning, Jilin, Guangdong, Sichuan and Hunan). An additional 200 unrelated healthy volunteers served as a control group. Routine screenings and complete ophthalmological examinations were performed on all participants following the provision of signed informed consent. The typical clinical features of OCA, including hypopigmented hair, skin and eyes, nystagmus, photophobia, poor vision and foveal hypoplasia, were observed 
Table I. Primers for amplification and sequence analysis of human TYR.

Primer

Primer sequence $\left(5^{\prime}-3^{\prime}\right)$

TYR-EXON1A-F

TYR-EXON1A-R

TYR-EXON1B-F

TYR-EXON1B-R

TYR-EXON2-F

TYR-EXON2-R

TYR-EXON3-F

TYR-EXON3-R

TYR-EX0N4-F

TYR-EX0N4-R

TYR-EXON5-F

TYR-EXON5-R
CCAGTTCCTGCAGACCTTGT
TCATTTGGCCATAGGTCCCT
GGGACCAAACTGCACAGAGA
GGCTGCAATGAGTGTTCAGG
TGATGGATTTCTCAGAACATATCCCT
ACAACACATATTCTTGGTCAACTCA
TGGGATAATCACATAGGTTTTCAGT
GGTGACAACCTGATCACAGACA
CCATGTCTCCAGATTTAATATATGCC
CACTTTCAGGATTTAAAGTGTTCAGGA
GCCTTCAAACCCAGGTGTCT
GGAACCTGGACATTACTTTGAGT

TYR, tyrosinase; F, forward; R, reverse.

in all patients. None of the patients had any other systemic diseases. The Institutional Review Board of the Tongji Eye Institute of Tongji University School of Medicine (Shanghai, China) approved the study, and all procedures were performed in accordance with the tenets of the Declaration of Helsinki.

Genetic analysis. Genomic DNA was extracted from peripheral leukocytes using the Tiangen RelaxGene Blood DNA system (Tiangen Biotech, Co., Ltd., Beijing, China) according to the manufacturer's protocol. The primers (Table I) were designed for all five exons and splice junction sites of the TYR gene using Primer3 software (version 0.4.0; http://bioinfo. ut.ee/primer3-0.4.0/). All exons and flank regions of TYR were amplified using polymerase chain reaction (PCR). A total of $25 \mu \mathrm{l}$ PCR mixture contain $40 \mathrm{ng}$ genomic DNA, $1 \mathrm{mM}$ each forward and reverse primers and $12.5 \mu 12 \mathrm{X}$ Taq PCR MasterMix (Tiangen Biotech Co., Ltd.). PCR was performed on C1000 Touch Thermal Cycler (Bio-Rad Laboratories, Inc., Hercules, CA, USA) using Touchdown PCR program with 35 cycles of amplification: $95^{\circ} \mathrm{C}$ for $30 \mathrm{sec} ; 64-57^{\circ} \mathrm{C}$ for $30 \mathrm{sec}$ beginning at $64^{\circ} \mathrm{C}$ and decreasing by $0.5^{\circ} \mathrm{C}$ each cycle for 14 cycles, until finishing at a final annealing temperature of $57^{\circ} \mathrm{C}$ for 21 cycles and at $72^{\circ} \mathrm{C}$ for $40 \mathrm{sec}$.

The PCR products were sequenced using an ABI3730 automated sequencer (PE Biosystems, Foster City, CA, USA). Benign polymorphisms were excluded using the database of the 1000 Genomes Project (http://www.1000genomes.org/) and the dbSNP National Center for Biotechnology Information database (http://www.ncbi.nlm.nih.gov/projects/SNP/). The albinism database (http://www.ifpcs.org/albinism/) and reported literature were then used to determine whether the mutations had been previously reported as pathogenic. To predict the pathogenicity of TYR variants in the present study, in silico analysis was performed with Sorting Intolerant From Tolerant (SIFT; http://sift.jcvi.org/),Polymorphism Phenotyping (PolyPhen)-2 (http://genetics.bwh.harvard.edu/pph2/), I-Mutant (http://folding.biofold.org/i-mutant/i-mutant2.0.html) and Human Splicing Finder (HSF) 3.0 (http://www.umd. be/HSF3/HSF.shtml), respectively. The conservation of the amino acid substitutions were assessed by multiple sequence alignment with sequences from Homo sapiens (NP_000363.1), Canis lupus familiaris (NP_001002941.1), Bos taurus (NP_851344.1), Mus musculus (NP_035791.1), Rattus norvegicus (NP_001101005.1), Gallus gallus (NP_989491.1), Danio rerio (NP_571088.1), Xeno pustropicalis (NP_001096518.1) and Bacillus megaterium (UniProtKB:B2ZB02). The crystal structure of protein data bank (PDB): 5I3B (TYR from Bacillus megaterium with configuration $\mathrm{B}$ of hydroquinone inhibitor in the active site) was used to predict and improve understanding of the potential impact of the novel mutation (8).

\section{Results}

In the present study, 17 patients were diagnosed with non-syndromic OCA based on the routine screenings and complete ophthalmological examination. The parents of all patients were unaffected, which is consistent with the pattern of autosome recessive inheritance. Using PCR combined with Sanger sequencing, a total of 12 mutations were found in TYR, including 10 previously reported mutations associated with OCA1 and two novel mutations (Fig. 1 and Table II). Patient 4000101 was a compound heterozygote for mutations c.1A>G (p.M1?) and c.896G >A (p.R299H) in the TYR gene. Patient 4000201 was a compound heterozygote for mutations c.1198T>G (p.W400G) and c.896G>A (p.R299H). Patient 4000301, from a consanguineous family, was a homozygote for mutation c.929_930insC (p.R311Kfs*7). Patient 4000701 was compound heterozygous for c.929_930insC (p.R311Kfs"7) and c.896G>A (p.R299H). Patient 4000801 was a homozygote for c.819G>T (p.Q273H). Patient 4000901 was a compound heterozygote for mutations c.758G $>A$ (p.G253E) and c.896G>A (p.R299H). Patient 4001101 was a compound heterozygote for mutations c.231_232insGGG (p.R77_E78insG) and c.230G >A (p.R77Q), and c.230G >A was confirmed in the unaffected family member of patient 4001101 (Fig. 1). Patient 4001301 carried mutations c.346C $>$ T (p.R116*) and c.832C>T (p.R278*), patient 4001501 carried mutations c.896G >A (p.R299H) and c.70T>C (p.C24R), 
Table II. Summary of TYR mutations in the present study.

\begin{tabular}{|c|c|c|c|c|c|c|c|c|}
\hline \multirow[b]{2}{*}{ Patient } & \multirow[b]{2}{*}{ Gender } & \multirow[b]{2}{*}{$\begin{array}{c}\text { Clinical } \\
\text { diagnosis }\end{array}$} & \multirow[b]{2}{*}{ Gene } & \multicolumn{2}{|c|}{ Mutation 1} & \multicolumn{2}{|c|}{ Mutation 2} & \multirow[b]{2}{*}{ Province } \\
\hline & & & & $\begin{array}{l}\text { Nucleotide } \\
\text { change }\end{array}$ & $\begin{array}{l}\text { Amino acid } \\
\text { change }\end{array}$ & $\begin{array}{l}\text { Nucleotide } \\
\text { change }\end{array}$ & $\begin{array}{l}\text { Amino acid } \\
\text { change }\end{array}$ & \\
\hline 4000101 & $\mathrm{~F}$ & OCA & TYR & c. $1 \mathrm{~A}>\mathrm{G}$ & p.M1? & c. $896 \mathrm{G}>\mathrm{A}$ & p.R299H & Jilin \\
\hline 4000201 & M & OCA & TYR & c. $1198 \mathrm{~T}>\mathrm{G}$ & p.W400G & c. $896 \mathrm{G}>\mathrm{A}$ & p.R299H & Jiangsu \\
\hline 4000301 & M & OCA & TYR & c.929_930insC & p.R311Kfs*7 & c.929_930insC & p.R311Kfs*7 & Jiangsu \\
\hline 4000701 & M & OCA & TYR & c.929_930insC & p.R311Kfs*7 & c. $896 \mathrm{G}>\mathrm{A}$ & p.R299H & Guangdong \\
\hline 4000801 & M & OCA & TYR & c. $819 \mathrm{G}>\mathrm{T}$ & p.Q273H & c. $819 \mathrm{G}>\mathrm{T}$ & p.Q273H & Guangdong \\
\hline 4000901 & $\mathrm{~F}$ & OCA & TYR & c. $758 \mathrm{G}>\mathrm{A}$ & p.G253E & c. $896 \mathrm{G}>\mathrm{A}$ & p.R299H & Sichuan \\
\hline 4001101 & $\mathrm{~F}$ & OCA & TYR & c.231_232insGGG & p.R77_E78insG & c. $230 \mathrm{G}>\mathrm{A}$ & p.R77Q & Sichuan \\
\hline 4001301 & M & OCA & TYR & c. $346 \mathrm{C}>\mathrm{T}$ & p.R116* & c. $832 \mathrm{C}>\mathrm{T}$ & p.R278* & Hunan \\
\hline 4001501 & M & OCA & TYR & c. $70 \mathrm{~T}>\mathrm{C}$ & p.C24R & c. $896 \mathrm{G}>\mathrm{A}$ & p.R299H & Beijing \\
\hline 4001701 & M & OCA & TYR & c.231_232insGGG & p.R77_E78insG & c. $1037-7 \mathrm{~T}>\mathrm{A}$ & - & Jiangsu \\
\hline
\end{tabular}

OCA, oculocutaneous albinism; TYR, tyrosinase; M, male; F, female.
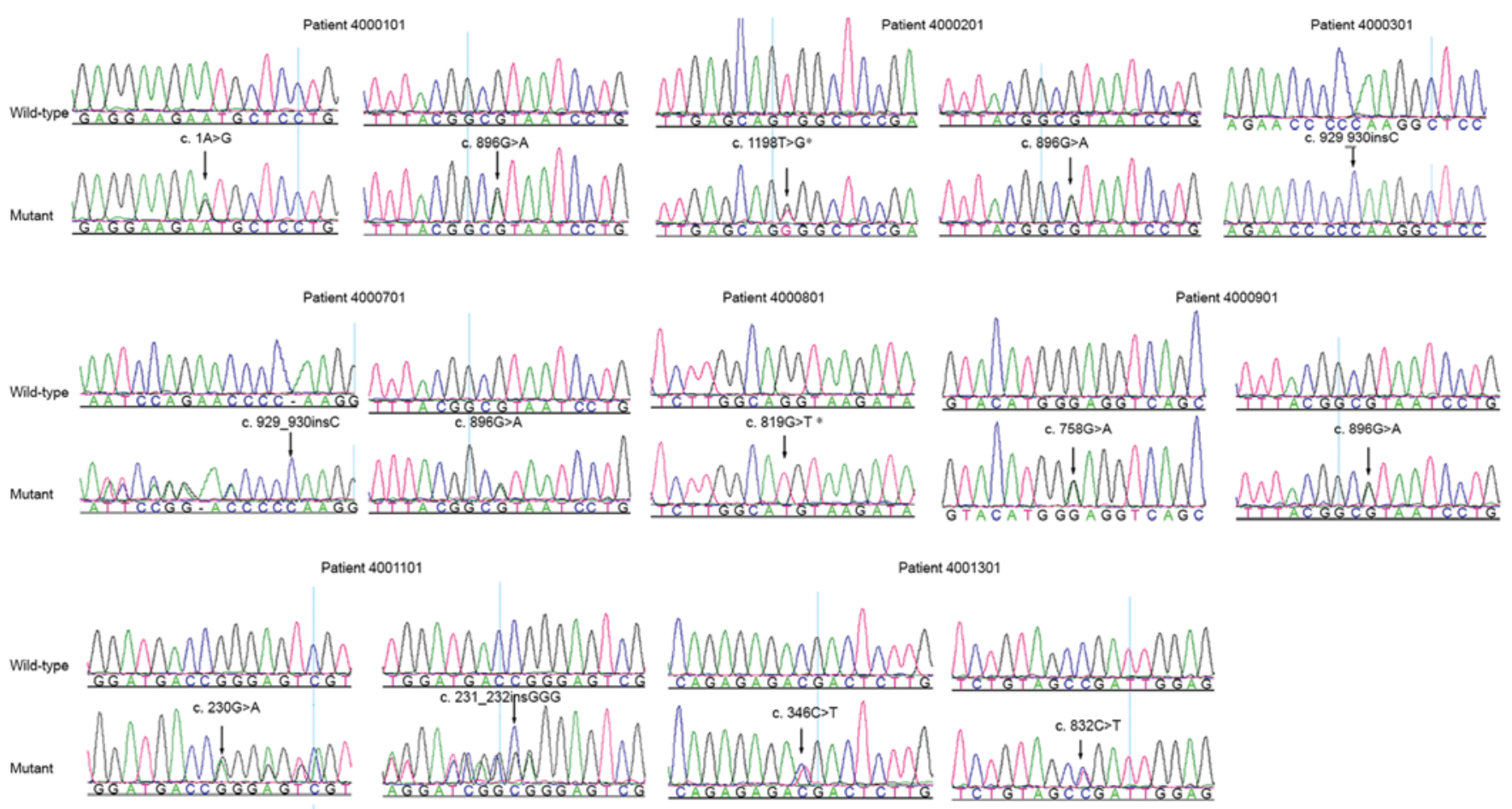

Patient 4001301

Patient 4001501

Patient 4001701

Individual 4001102
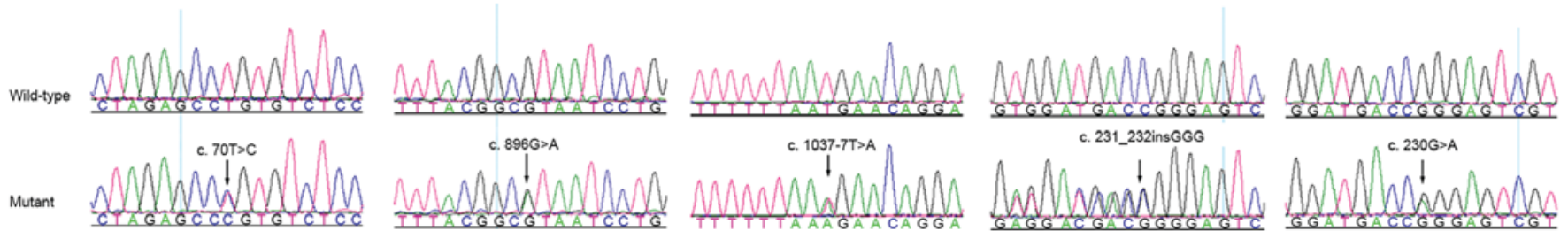

Figure 1. Sequence electropherograms of TYR mutations and wild-type TYR identified in patients with OCA. Arrows indicate mutations in the index patients, respectively. The asterisk indicates novel mutations associated with OCA for the first time. Individual 4001102 (Patient 4001101 's brother) was unaffected, whose sequence electropherogram results were used to confirm the c.230G>A (p.R77Q) mutation identified in Patient 4001101. OCA, oculocutaneous albinism; TYR, tyrosinase.

and patient 4001701 carried the mutation c.231-232insGGG (p.R77_E78insG) and the splicing mutation c.1037-7T>A.
Among the mutations identified, two mutations c.819G $>$ T (p.Q273H) and c.1198T>G (p.W400G) were novel, and their 


$\begin{array}{lll}\text { A } & & 400 \\ \text { H.sapiens } & 390 & \text { HAFVDSIFEQWLRRHRPLQEVYP } \\ \text { P.troglodytes } & 390 & \text { HAFVDSIFEQWLRRHRPLQEVYP } \\ \text { M.mulatta } & 390 & \text { HAFVDSIFEQWLRRHHPLQEVYP } \\ \text { C.lupus } & 390 & \text { HAFVDSIFEQWLRRHHPLREVYP } \\ \text { B.taurus } & 390 & \text { HAFVDSIFEQWLRKYHPLQDVYP } \\ \text { M.musculus } & 390 & \text { HAFVDSIFEQWLRRHRPLLVYP } \\ \text { R.norvegicus } & 390 & \text { HAFVDSIFEQWLRRHRPLLEVYP } \\ \text { G.gallus } & 390 & \text { HAFVDSIFERWLRRHRPMLEYP } \\ \text { D.rerio } & 392 & \text { HAFIDSIFEQWLRRHQPPRTHP } \\ \text { X.tropicalis } & 394 & \text { HAFVDSIFEQWLRRHGASVDIYP } \\ \text { B. megaterium } & 231 & \text { HANVDRIWAVWQIIHRN-QNYQP } \\ & & *\end{array}$

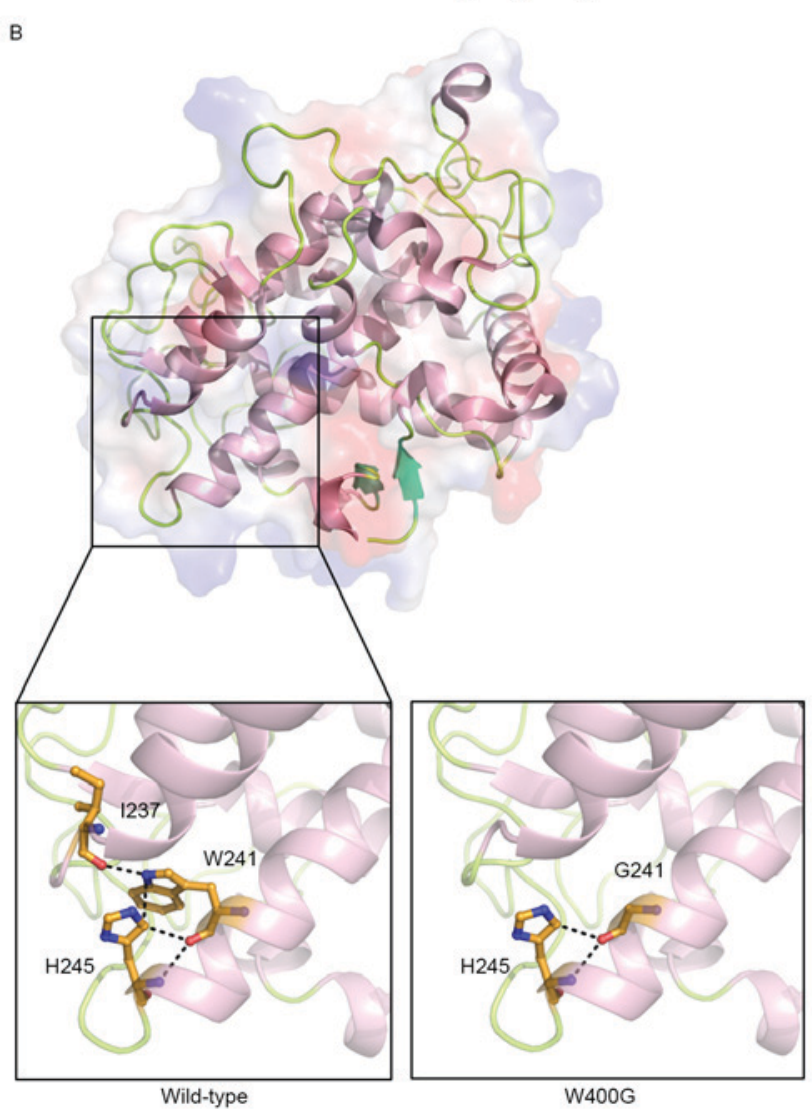

Figure 2. Effect of the identified mutation found in $\mathrm{W}^{400}$ of TYR (A) Alignment of multiple TYR amino acid sequences across species. The amino acid of $\mathrm{W}^{400}$ (shaded gray) was completely conserved from bacteria to humans. The amino acid residue $\mathrm{W}^{241}$ interacts with $\mathrm{I}^{237}$ and $\mathrm{H}^{245}$ in TYR of Bacillus megaterium. The asterisk indicates the three residues. (B) Analysis of the crystal structure of TYR (from PDB ID: 5I3B) and the predicted structure of W241G from Bacillus megaterium. (Left, wild-type; right, predicted structure of bacterial W241G mutant to determine the effect of human W400G. Comparison of the structures between the wild-type and the mutant showed $\mathrm{W}^{241}$ interacted with $\mathrm{I}^{237}$ and $\mathrm{H}^{245}$ in the wild-type, whereas this substitution in $\mathrm{W} 241 \mathrm{G}$ disrupted this interaction, possibly impairing TYR structure. TYR, tyrosinase.

pathogenicity were predicted via in silico analysis. The SIFT and PolyPhen-2 scores for the c.819G >T (p.Q273H) mutation were 0.030 and 0.981 , respectively, which predicted that the mutation was 'probably damaging'. The I-Mutant online server predicted that the mutation may decrease protein stability with a reliability index (RI) of 6 . The c.1198T $>$ G mutation in the TYR gene was not detected in the 200 normal controls enrolled in the present study, nor was it present within the dbSNP database, the 1000 Genomes Project or the albinism database (http://www.ifpcs.org/albinism/). This mutation, resulting in the conversion from tryptophan to glycine at

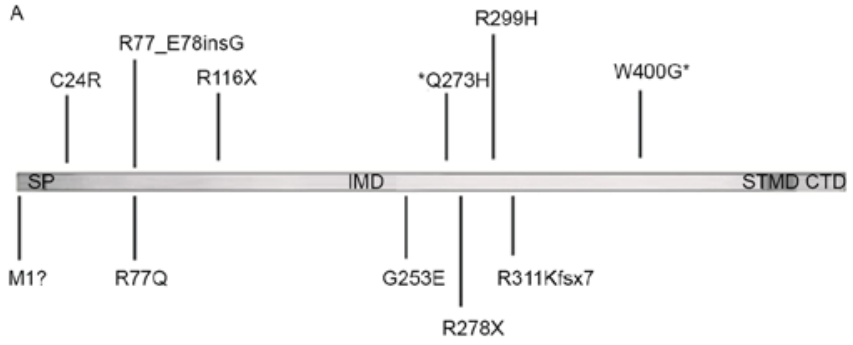

B

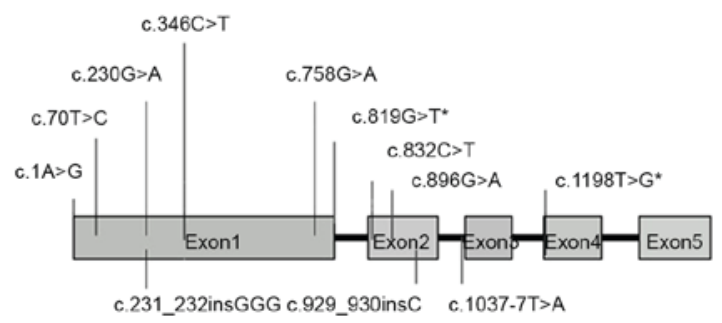

Figure 3. Diagrams of the linear location of identified TYR mutations (A) Locations of the identified mutations of the genomic DNA in TYR. The asterisk indicates novel mutations associated with oculocutaneous albinism for the first time. (B) Schematic diagram showing the location of the identified mutations in TYR protein. SP, signal peptide; IMD, intramelanosomal domain; STMD, single trans-membrane domain; CTD, C-terminal domain; TYR, tyrosinase.

codon 400, was predicted to be 'probably damaging' with a score of 0.99 by PolyPhen 2 and a score of 0 by SIFT, and it was predicted that the protein stability may decrease with an RI of 9 by the I-Mutant online server. Multiple sequence alignment showed that residues $\mathrm{W}^{241}, \mathrm{I}^{237}$ and $\mathrm{H}^{245}$ in the TYR protein of Bacillus megaterium are highly conserved across species, and correspond to $\mathrm{W}^{400}, \mathrm{I}^{393}$ and $\mathrm{H}^{404}$ in the human TYR protein, respectively (Fig. 2A). The crystal structure of wild-type TYR (PDB ID: 5I3B) showed that $\mathrm{W}^{241}$ interacts with $\mathrm{I}^{237}$ and $\mathrm{H}^{245}$, and this interaction may be impaired when $\mathrm{W}^{241}$ is substituted by amino acid G (Fig. 2B).

\section{Discussion}

TYR is a glycoprotein containing four regions, a signal sequence (SP; amino acid residues 1-18), an intramelanosomal domain (IMD; residues 19-476) with a binuclear copper binding site, a single $\alpha$-helical trans-membrane domain (STMD; residues 477-497), and a flexible C-terminal domain (CTD; residues 498-529), as shown in Fig. 3A (9). There are three key enzymes, TYR, TYRP1 and dopachrome tautomerase, which are important in melanin biosynthesis (10). Among these, a defect in TYR can lead to the complete or partial lack of melanin as TYR catalyzes the critical first and second reactions in the conversion of TYR to melanin (11). The human TYR gene located on chromosome 11q14.3 is composed of five exons encoding a copper binding protein with a molecular weight of $\sim 75 \mathrm{kDa}$. In the present study, mutation analysis of the TYR gene was performed in 17 patients with OCA, which identified 12 mutations as the cause of OCA in 10 of the patients with OCA. Of these mutations, 10 mutations were clustered in exon 1 and exon 2, which are the mutation hotspots of TYR in the Chinese Han population (5). For the additional two mutations, one mutation c.1198T $>\mathrm{G}$ (p.W400 G) was located in exon 4 and another mutation IVS2-7T $>A$ was located in intron 2 (Fig. 3B). In Chinese patients with OCA1, missense 
mutations of TYR have been found to account for almost $62.5 \%$ of all reported TYR mutations, whereas the insertion and deletion mutations account for $25 \%$ (12).

Of the 12 mutations identified in the present study, 10 mutations were reported as causative for OCA in previous literature. Among these 10 mutations, R299H was the most frequent mutation in the present study (5/12). R299H is the most frequent mutation in Chinese, Caucasian, Korean, and Christian Arab populations, and this mutation may affect the function of TYR by disrupting copper binding and different substitutions occurring in $\mathrm{R}^{299}(5,13-15)$. c.231-232insGGG is another frequent mutation in the Chinese OCA1 population, resulting in an insertion of glycine residue between $\mathrm{R}^{77}$ and $\mathrm{E}^{78}$ (13). Mutation c. $230 \mathrm{G}>\mathrm{A}$, resulting in an amino acid change from arginine to glutamine at position 77 , has been reported in the Japanese, Korean, Chinese, German and Pakistani populations (16). The mutation c.929_930insC, resulting in a truncated polypeptide, is the most frequent mutation allele in far East Asian OCA1 populations $(13,17)$. The missense mutation c.1A>G (p.M1?) has been found in British and Chinese patients with OCA1, and this may alter codons initiating translation to M31, resulting in a skipping of the putative SP $(18,19)$. The splicing mutation IVS2-7T $>A$ is frequently found in patients with OCA1 worldwide and can result in pathological splicing sites (19-22).

It is the first time, to the best of our knowledge, that the additional two mutations c.819G $>$ T (p.Q273H) and c.1198T >G (p.W400G) identified in the present study have been associated with OCA. In the in silico analysis, c.819G >T (p.Q273H) was predicted to be pathogenic, although it has been reported as rs748669377 with a frequency of 1:120,054 in the Exome Aggregation Consortium (http://exac.broadinstitute.org/). In addition, HSF 3.0 predicted that this mutation may result in disruption of the wild-type donor site, most likely affecting splicing. However, its exact mechanism requires further investigation for confirmation. The other novel mutation c.1198T >G (p.W400G) occurred at the same amino acid position as another pathogenic mutation c.1199G $>$ T (p.W400L). The amino acid residue $\mathrm{W}^{400}$ locating in the IMD of the human TYR protein, was found to be highly conserved from bacteria to humans (Fig. 2A). Residues $\mathrm{W}^{241}, \mathrm{I}^{237}$ and $\mathrm{H}^{245}$ in the TYR protein of Bacillus megaterium corresponded with $\mathrm{W}^{400}, \mathrm{I}^{393}$ and $\mathrm{H}^{404}$ in the human TYR protein, respectively (Fig. 2A). Structural analysis of wild-type TYR from Bacillus megaterium (PDB: 5I3B) showed that $\mathrm{W}^{241}$ interacts with $\mathrm{I}^{237}$ and $\mathrm{H}^{245}$, and the predicted structure of the mutated protein shows that this substitution disrupted hydrogen bonds in these two amino acids, impairing the spatial conformation of TYR (Fig. 2B).

In conclusion, mutation analysis of the TYR gene in 17 patients with non-syndromic OCA revealed that 12 mutations of TYR may have caused OCA in 10 patients. Among these mutations, two novel mutations c.1198T>G (p.W400G) and c.819G $>$ T (p.Q273H) were identified, expanding on the mutation spectrum of TYR in OCA. This may assist in the prenatal examination and genetic diagnosis of OCA, and offers novel insight into the molecular mechanism of OCA1.

\section{Acknowledgements}

This study was supported by the National Key Basic Research Program of China (973 Program; grant no. 2015CB964601), the National Natural Science Foundation of China (grant no. 81371062). Professor Jianjun Chen was supported by the Thousand Youth Talents Program of China.

\section{References}

1. Kamaraj B and Purohit R: Mutational analysis of oculocutaneous albinism: A compact review. Biomed Res Int 2014: 905472, 2014.

2. Summers CG: Albinism: Classification, clinical characteristics, and recent findings. Optom Vis Sci 86: 659-662, 2009.

3. Gronskov K, Brøndum-Nielsen K, Lorenz B and Preising MN: Clinical utility gene card for: Oculocutaneous albinism. Eur J Hum Genet 22, 2014.

4. Gong Y, Shao C, Zheng H, Chen B and Guo Y: Study on genetic epidemiology of albinism. Yi Chuan Xue Bao 21: 169-172, 1994 (In Chinese).

5. Wei A, Wang Y, Long Y, Wang Y, Guo X, Zhou Z, Zhu W, Liu J, Bian X, Lian S and Li W: A comprehensive analysis reveals mutational spectra and common alleles in Chinese patients with oculocutaneous albinism. J Invest Dermatol 130: 716-724, 2010.

6. Grønskov K, Ek J and Brondum-Nielsen K: Oculocutaneous albinism. Orphanet J Rare Dis 2: 43, 2007.

7. Kausar T, Bhatti MA, Ali M, Shaikh RS and Ahmed ZM: OCA5, a novel locus for non-syndromic oculocutaneous albinism, maps to chromosome 4q24. Clin Genet 84: 91-93, 2013.

8. Deri B, Kanteev M, Goldfeder M, Lecina D, Guallar V, Adir N and Fishman A: The unravelling of the complex pattern of tyrosinase inhibition. Sci Rep 6: 34993, 2016.

9. Lai X, Soler-Lopez M, Wichers HJ and Dijkstra BW: Large-scale recombinant expression and purification of human tyrosinase suitable for structural studies. PLoS One 11: e0161697, 2016.

10. Yamaguchi Y and Hearing VJ: Melanocytes and their diseases. Cold Spring Harb Perspect Med 4: pii: a017046, 2014.

11. Sánchez-Ferrer A, Rodríguez-López JN, García-Cánovas F and García-Carmona F: Tyrosinase: A comprehensive review of its mechanism. Biochim Biophys Acta 1247: 1-11, 1995.

12. Liu J, Choy KW, Chan LW, Leung TY, Tam PO, Chiang SW, Lam DS, Pang CP and Lai TY: Tyrosinase gene (TYR) mutations in Chinese patients with oculocutaneous albinism type 1. Clin Exp Ophthalmol 38: 37-42, 2010.

13. Tsai CH, Tsai FJ, Wu JY, Lin SP, Chang JG, Yang CF and Lee CC: Insertion/deletion mutations of type I oculocutaneous albinism in chinese patients from Taiwan. Hum Mutat 14: 542, 1999.

14. Tripathi RK, Strunk KM, Giebel LB, Weleber RG and Spritz RA: Tyrosinase gene mutations in type I (tyrosinase-deficient) oculocutaneous albinism define two clusters of missense substitutions. Am J Med Genet 43: 865-871, 1992.

15. Park SK, Lee KH, Park KC, Lee JS, Spritz RA and Lee ST: Prevalent and novel mutations of the tyrosinase gene in Korean patients with tyrosinase-deficient oculocutaneous albinism. Mol Cells 7: 187-191, 1997.

16. Shah SA, Raheem N, Daud S, Mubeen J, Shaikh AA, Baloch AH, Nadeem A, Tayyab M, Babar ME and Ahmad J: Mutational spectrum of the TYR and SLC45A2 genes in Pakistani families with oculocutaneous albinism, and potential founder effect of missense substitution (p.Arg77Gln) of tyrosinase. Clin Exp Dermatol 40: 774-780, 2015.

17. Park SH, Chae H, Kim Y and Kim M: Molecular analysis of Korean patients with oculocutaneous albinism. Jpn J Ophthalmol 56: 98-103, 2012.

18. Liu N, Kong XD, Shi HR, Wu QH and Jiang M: Tyrosinase gene mutations in the Chinese Han population with OCA1. Genet Res (Camb) 96: e14, 2014.

19. Breimer LH, Winder AF, Jay B and Jay M: Initiation codon mutation of the tyrosinase gene as a cause of human albinism. Clin Chim Acta 227: 17-22, 1994.

20. Ko JM, Yang JA, Jeong SY and Kim HJ: Mutation spectrum of the TYR and SLC45A2 genes in patients with oculocutaneous albinism. Mol Med Rep 5: 943-948, 2012.

21. King RA, Pietsch J, Fryer JP, Savage S, Brott MJ, Russell-Eggitt I, Summers CG and Oetting WS: Tyrosinase gene mutations in oculocutaneous albinism 1 (OCA1): Definition of the phenotype. Hum Genet 113: 502-513, 2003.

22. Goto M, Sato-Matsumura KC, Sawamura D, Yokota K, Nakamura $\mathrm{H}$ and Shimizu H: Tyrosinase gene analysis in Japanese patients with oculocutaneous albinism. J Dermatol Sci 35: 215-220, 2004. 